\title{
Diferenciação de lotes de sementes de quiabo pelo teste de envelhecimento acelerado
}

\author{
Differentiation of seeds lots of okra by accelerated aging test
}

\author{
Salvador Barros Torres ${ }^{{ }^{*}}$ Francisca Gleiciane da Silva ${ }^{\mathrm{I}}$ Maryjane Diniz de Araújo Gomes ${ }^{\mathrm{I}}$ \\ Clarisse Pereira Benedito ${ }^{I}$ Francisco Elder Carlos Bezerra Pereira ${ }^{I}$ Edvan Costa da Silva ${ }^{I}$
}

\section{RESUMO}

Os testes de germinação e de vigor são componentes essenciais do processo de controle de qualidade das empresas produtoras de sementes, e o teste de envelhecimento acelerado é um dos mais utilizados para avaliação do vigor de sementes. Nesse sentido, a pesquisa teve como objetivo estudar a metodologia do teste de envelhecimento acelerado, visando à identificação de diferentes níveis de vigor de lotes de sementes de quiabo (Abelmoschus esculentus (L.) Moench). Para isso, quatro lotes de sementes da cultivar 'Colhe Bem'e cinco lotes da cultivar 'Santa Cruz 47' foram submetidos às seguintes avaliações: germinação, emergência de plântulas, envelhecimento acelerado tradicional e em solução saturada com $\mathrm{NaCl}\left(38\right.$ e $41^{\circ} \mathrm{C}$ durante 24, 48, 72 e 96 horas) e grau de umidade no início dos testes e após a incubação para o teste de envelhecimento acelerado. Os testes de envelhecimento acelerado tradicional e em solução saturada com cloreto de sódio, a $41^{\circ} \mathrm{C}$ durante 96 horas, constituem em opções promissoras para detectar diferenças de vigor entre lotes de sementes de quiabo.

Palavras-chave: Abelmoschus esculentus, Malvaceae, vigor, potencial fisiológico.

\section{ABSTRACT}

The vigor and germination tests are essential components of seed quality control for the seed industry. Accelerated aging is one for the most useful tests used for the evaluation of seed vigor. In this sense, this paper aimed to study the methodology of the accelerated aging test, in order to identify different levels of vigor seed lots of okra (Abelmoschus esculentus (L.) Moench). Four seed lots of the 'Colhe Bem' cultivar and five seed lots of the 'Santa Cruz 47' were submitted to the following assessments: germination, seedling emergence, accelerated aging by traditional procedures and saturated $\mathrm{NaCl}\left(38\right.$ and $41^{\circ} \mathrm{C}$ for 24, 48, 72 and 96 hours) and moisture content at the beginning of the tests and, after incubation to accelerated aging test. The accelerated aging test at $41^{\circ} \mathrm{C} / 96$ hours (traditional procedure and saturated $\mathrm{NaCl}$ ) consisted efficient to detect vigor differences among seed lots of okra.

Key words: Abelmoschus esculentus, Malvaceae, vigor, physiological potential.

\section{INTRODUÇÃO}

O quiabo (Abelmoschus esculentus (L.) Moench) é uma cultura muito apropriada à agricultura familiar, especialmente, devido aos gastos com mãode-obra nas operações de colheita, classificação e embalagem, cujas cultivares mais empregadas são 'Colhe Bem' e 'Santa Cruz 47' (FILGUEIRA, 2008). Trata-se de uma hortaliça fruto adaptada a climas tropicais e subtropicais, amplamente cultivada no Brasil e muito importante sob o ponto de vista econômico (PURQUERIO et al., 2010), mas pouco contemplada pela pesquisa, principalmente na área de sementes.

A utilização de sementes de alta qualidade constitui a base para aumento da produtividade agrícola, o que é objeto de inúmeras pesquisas com outras espécies cultivadas, é ainda pouco pesquisado para algumas olerícolas (TUNES et al., 2011). Nesse sentido, segundo KIKUTI \& MARCOS FILHO (2012), os testes de vigor se constituem em ferramentas importantes para complementação das informações obtidas no teste de germinação.

Dos testes de vigor utilizados para avaliar a qualidade das sementes, o envelhecimento aceleradoé

IDepartamento de Ciências Vegetais (DCV), Universidade Federal Rural do Semi-Árido (UFERSA), 59625-900, Mossoró, RN, Brasil. E-mail: sbtorres@ufersa.edu.br. *Autor para correspondência. 
capaz de proporcionar informações consistentes com desempenho de lotes de sementes no campo e após o armazenamento (KIKUTI \& MARCOS FILHO, 2012). Esse teste acelera artificialmente a taxa de deterioração das sementes através de sua exposição a níveis elevados de temperatura e umidade relativa do ar, considerados os fatores ambientais preponderantes na intensidade e velocidade de deterioração (MARCOS FILHO, 1999). Nessa situação, sementes de baixa qualidade deterioram-se mais rapidamente do que as mais vigorosas, apresentando queda diferenciada da viabilidade (PEREIRA et al., 2012).

Dentre os fatores que afetam $O$ comportamento das sementes submetidas ao teste de envelhecimento acelerado, a interação temperatura/ período de exposição é um dos mais estudados. Nesse sentido, há algumas indicações na literatura para a condução do teste de envelhecimento acelerado tradicional, com a incubação a 144 horas a $41^{\circ} \mathrm{C} \mathrm{em}$ sementes de quiabo (TORRES \& CARVALHO, 1998) e 48 horas a $41^{\circ} \mathrm{C}$, em sementes de alface e almeirão (SANTOS et al., 2011). Para o envelhecimento acelerado em solução salina saturada, existem algumas recomendações, como a incubação a 48 e 72 horas a $42^{\circ} \mathrm{C}$ ou 48 horas a $41^{\circ} \mathrm{C}$, em sementes de coentro (PEREIRA et al., 2011; TUNES et al., 2011); 72 horas a $41^{\circ} \mathrm{C}$, em sementes de alface e almeirão (SANTOS et al., 2011); 48 horas a $41^{\circ} \mathrm{C}$, em sementes de jiló (LOPES et al., 2012); e 48 e 72 horas a $41^{\circ} \mathrm{C}$, em sementes de alface (KIKUTI \& MARCOS FILHO, 2012).

No que se refere às sementes de quiabo, as metodologias para avaliação do vigor são bastante escassas. Até o momento, é verificada apenas a eficiência do teste de deterioração controlada, conduzido a $45^{\circ} \mathrm{C}$, com grau de umidade das sementes de $24 \%$ e período de exposição de 24 horas (TORRES et al., 2013); e, para o teste de envelhecimento, o período de 144 horas, a $41^{\circ} \mathrm{C}$, mostrou-se eficiente na separação dos lotes em diferentes níveis de vigor (TORRES \& CARVALHO, 1998). Assim, objetivouse, nesta pesquisa determinar metodologias para a condução do teste de envelhecimento acelerado, visando à identificação de diferentes níveis de vigor em lotes de sementes de quiabo.

\section{MATERIAL E MÉTODOS}

O experimento foi realizado no Laboratório de Análise de Sementes do Departamento de Ciências Vegetais da UFERSA, durante o período de agosto de 2011 a maio de 2012, utilizando as cultivares de quiabo 'Colhe Bem' (quatro lotes) e 'Santa Cruz 47' (cinco lotes), provenientes de empresas nacionais, produzidas no ano agrícola de 2010/2011. Durante a fase experimental, as sementes permaneceram acondicionadas em sacos de papel multifoliado e mantidas em ambiente controlado $\left(15^{\circ} \mathrm{C}\right.$ e $40 \%$ de umidade relativa do ar).

Os lotes foram avaliados por meio dos seguintes testes e/ou determinações: a) germinação: quatro subamostras de 50 sementes foram semeadas em caixas de acrílico transparente $(11 \times 11 \times 3 \mathrm{~cm})$, tendo como substrato areia lavada e esterilizada, umedecida inicialmente com quantidade de água equivalente a $60 \%$ da capacidade de retenção e colocadas para germinar em câmara do tipo Biological Oxygen Demand (B.O.D.), a $20-30^{\circ} \mathrm{C}$, na ausência de luz. As avaliações foram realizadas aos quatro e 21 dias após a semeadura, com os resultados expressos em porcentagem de plântulas normais (BRASIL, 2009); b) emergência de plântulas em campo: utilizouse quatro subamostras de 50 sementes por lote que foram semeadas em canteiros, na profundidade de $2 \mathrm{~cm}$. A avaliação foi realizada aos 21 dias após a semeadura, mediante a contagem de plantulas normais emergidas. Os resultados foram expressos em porcentagem média de emergência de plântulas para cada lote; c) envelhecimento acelerado tradicional: conduzido utilizando-se caixas plásticas transparentes $(11 \times 11 \times 3 \mathrm{~cm})$, tipo gerbox, contendo $40 \mathrm{~mL}$ de água destilada. As sementes foram dispostas sobre a tela metálica existente no interior da caixa plástica e tampada. Em seguida, as amostras de sementes foram mantidas em incubadora a $38^{\circ} \mathrm{C}$ e $41^{\circ} \mathrm{C}$, durante 24 , 48, 72 e 96 horas. Decorrido esse período, quatro amostras de 50 sementes por lote foram colocadas para germinar, utilizando o mesmo procedimento adotado para o teste de germinação. Avaliou-se aos quatro dias após a semeadura, computando-se a porcentagem de plântulas normais (BRASIL, 2009); e) envelhecimento acelerado em solução saturada com $\mathrm{NaCl}$ : realizado conforme descrito para o teste de envelhecimento acelerado tradicional, com exceção de serem adicionados ao fundo de cada caixa plástica $40 \mathrm{~mL}$ de solução saturada de cloreto de sódio (40g de $\mathrm{NaCl}$ em $100 \mathrm{~mL}$ de água), visando a proporcionar ambiente com $76 \%$ de umidade relativa do ar (JIANHUA \& McDONALD, 1997); f) grau de umidade: determinado antes do início dos testes e, após a incubação, para o teste de envelhecimento acelerado. Para isso, utilizou-se o método da estufa (BRASIL, 2009), empregando-se duas repetições de aproximadamente quatro gramas de sementes, a $105 \pm 3^{\circ} \mathrm{C}$ por 24 horas, com resultados expressos em porcentagem média para cada lote. 
O delineamento experimental utilizado foi o inteiramente casualizado, com quatro repetições de 50 sementes, separadamente para cada teste e cultivar. Os dados foram submetidos à análise de variância e as médias foram comparadas pelo teste de Tukey, a $5 \%$ de probabilidade. Também foram calculados os coeficientes de correlação simples de Pearson (r) entre os resultados dos testes de envelhecimento acelerado e os de emergência de plântulas em campo, sendo a significância dos valores de $\mathrm{r}$ determinada pelo teste $\mathrm{t}$ a $1 \%$ e $5 \%$ de probabilidade.

\section{RESULTADOS E DISCUSSÃO}

Os valores referentes aos graus de umidade inicial das sementes de quiabo foram semelhantes para os nove lotes estudados, variando de 11,1 a $12,0 \%$ ('Colhe Bem') e 11,2 a $13,3 \%$ ('Santa Cruz 47') (Tabela 1). Essa variação, relativamente pequena, indica que não houve interferência do grau de umidade das sementes nos resultados obtidos nos testes de vigor. Conforme enfatiza MARCOS FILHO (1999), a uniformização dessa variável é imprescindível para a padronização das avaliações e obtenção de resultados consistentes.

A germinação dos lotes das duas cultivares foi superior à mínima estabelecida $(\geq 80 \%)$ para comercialização de sementes de quiabo. Não foram observadas diferenças entre os lotes das cultivares quanto à sua germinação (Tabela 1). Segundo MARCOS FILHO (1999), é importante a comparação de lotes de sementes com germinação semelhante e, de acordo com POWELL (1986), preferencialmente situados na fase I da curva de perda de viabilidade. Neste estudo, todos os lotes apresentaram alta qualidade, com germinação variando entre 88 a $96 \%$, estando situados na fase I da curva de perda de viabilidade de semente, caracterizada por ser relativamente longa e com poucas sementes mortas.

Quando as sementes de quiabo foram avaliadas por meio da primeira contagem de germinação, foi possível observar diferenças no vigor dos lotes (Tabela 1). Neste caso, os lotes 4 e 5 foram os mais vigorosos; já os lotes 7 e 9 da cultivar 'Santa Cruz 47' foram indicados como de baixo vigor e o lote 6 como intermediário, não diferindo do lote 8 . Pode ser verificado também que os lotes 1 e 3 da cultivar 'Colhe Bem' possuem baixa qualidade fisiológica, porém não diferindo do lote 2 . A maior sensibilidade da primeira contagem do teste de germinação em detectar diferenças entre lotes de sementes foi, também, confirmada por TORRES et al. (2012), quando avaliaram diferentes lotes de sementes de pepino e coentro. Segundo MEDEIROS et al. (2014), a primeira contagem do teste de germinação, muitas vezes, expressa melhor as diferenças de velocidade de germinação entre lotes do que os índices de velocidade de germinação. Ainda, segundo os mesmos autores, trata-se de uma avaliação menos trabalhosa que o de velocidade de germinação, além de ser conduzida simultaneamente com o teste de germinação, não exigindo equipamento especial.

Tabela 1 - Grau de umidade inicial, germinação, primeira contagem e emergência de plântulas de lotes de sementes de quiabo (Abelmoschus esculentus (L.) Moench), cultivares 'Colhe Bem' e 'Santa Cruz 47'.

\begin{tabular}{|c|c|c|c|c|c|}
\hline Cultivares & Lotes & Grau de umidade & Germinação & Primeira contagem & Emergência de plântulas \\
\hline \multirow{7}{*}{ 'Colhe Bem' } & & & & - & - \\
\hline & 1 & 12,0 & $90 \mathrm{a}$ & $18 \mathrm{~b}$ & $72 b$ \\
\hline & 2 & 11,7 & $91 \mathrm{a}$ & $23 \mathrm{ab}$ & $76 \mathrm{a}$ \\
\hline & 3 & 11,1 & $88 \mathrm{a}$ & $12 \mathrm{~b}$ & $70 \mathrm{~b}$ \\
\hline & 4 & 12,0 & $91 \mathrm{a}$ & $33 \mathrm{a}$ & $75 \mathrm{a}$ \\
\hline & $\mathrm{CV}(\%)$ & & 6,82 & 28,23 & 3,79 \\
\hline & $\mathrm{F}$ & & $1,82^{\mathrm{ns}}$ & $9,14^{*}$ & $11,38^{*}$ \\
\hline \multirow{7}{*}{ 'Santa Cruz 47' } & 5 & 12,0 & 93 a & $35 \mathrm{a}$ & $85 \mathrm{a}$ \\
\hline & 6 & 12,0 & $92 \mathrm{a}$ & $21 \mathrm{~b}$ & $81 \mathrm{a}$ \\
\hline & 7 & 11,6 & $91 \mathrm{a}$ & $14 \mathrm{c}$ & $72 b$ \\
\hline & 8 & 13,3 & 96 a & $29 a b$ & $88 \mathrm{a}$ \\
\hline & 9 & 11,2 & $95 \mathrm{a}$ & $13 \mathrm{c}$ & $75 \mathrm{~b}$ \\
\hline & $\mathrm{CV}(\%)$ & & 6,82 & 12,70 & 5,35 \\
\hline & $\mathrm{F}$ & & $0,82^{\mathrm{ns}}$ & $95,5^{*}$ & $11,40^{*}$ \\
\hline
\end{tabular}

Médias seguidas de mesma letra na coluna, para cada cultivar, não diferem entre si pelo teste de Tukey, a $5 \%$.

Ciência Rural, v.44, n.12, dez, 2014. 
Os lotes 2, 4, 5 e 8, quando avaliados pela emergência de plântulas em campo, foram mais vigorosos do que os lotes 1, 3, 7 e 9. Estes resultados coincidem com aqueles observados na germinação e primeira contagem de germinação (Tabela 1). Segundo MARCOS FILHO (1999), o teste de emergência de plântulas em campo constitui parâmetro indicador da eficiência dos testes para avaliação do potencial fisiológico de lotes de sementes.

Teste de envelhecimento acelerado tradicional, utilizando a temperatura de $38^{\circ} \mathrm{C}$ e períodos de 24, 48, 72 e 96 horas de condicionamento (Tabela 2), identificou diferenças de vigor entre os lotes de sementes de quiabo, sendo os lotes 1, 2 e 6 mais vigorosos e o lote 4 o de mais baixo vigor. Já utilizando à temperatura de $41^{\circ} \mathrm{C}$ (Tabela 3 ), os lotes 2,5 e 8 foram indicados como de alto vigor, enquanto os lotes 7 e 9 de baixo vigor. O vigor, comparando as duas temperaturas em que as sementes foram submetidas ao teste de envelhecimento acelerado dos lotes, foi melhor ajustado quando se utilizou à temperatura de $41^{\circ} \mathrm{C}$ durante o período de 96 horas de incubação. Os resultados desta combinação foram mais coerentes com os proporcionados pelo teste de emergência de plântulas e pela avaliação da primeira contagem do teste de germinação (Tabela 3). Nesse sentido, PEREIRA et al. (2011), para sementes de coentro, também recomendaram a mesma temperatura e período de incubação.

Para o envelhecimento acelerado em solução saturada com $\mathrm{NaCl}$ (Tabelas 4 e 5), de uma maneira geral, foi verificado que os resultados entre as duas metodologias utilizadas para envelhecer as sementes de quiabo artificialmente foram semelhantes. Porém, quando submetidos à temperatura de $41^{\circ} \mathrm{C}$ por 96 horas de incubação, os resultados obtidos quando as sementes foram envelhecidas em solução saturada de $\mathrm{NaCl}$ foram semelhantes aos verificados para o teste de emergência de plântulas em campo, primeira contagem de germinação e teste de envelhecimento acelerado tradicional, a $41^{\circ} \mathrm{C}$, utilizando-se o mesmo período de incubação. Resultados semelhantes também foram verificados por PEREIRA \& MARTINS FILHO (2010) para sementes de cubiu.

Com relação ao grau de umidade das sementes, após os períodos de envelhecimento tradicionais, constatou-se variação acentuada, atingindo valores entre 25,7 a $31,0 \%\left(38^{\circ} \mathrm{C}\right)$ e 22,6 a $30,4 \%\left(41^{\circ} \mathrm{C}\right)$ para os lotes da cultivar 'Colhe Bem'; 21,1 a $31,7 \%\left(38^{\circ} \mathrm{C}\right)$ e 21,6 a $32,6 \%\left(41^{\circ} \mathrm{C}\right)$ para os da cultivar 'Santa Cruz 47'. Esses valores superaram o limite de 3 a $4 \%$ de variação no grau de umidade, entre os lotes, após o período de incubação do teste de envelhecimento acelerado, de acordo com o recomendado por MARCOS FILHO (1999). Essa variação no grau de umidade das sementes foi devida, provavelmente, ao tamanho das sementes de quiabo, considerada relativamente pequena, portanto, podendo comprometer os resultados. Variações maiores no grau de umidade também foram observadas por MARTINS et al. (2006) para sementes de tomate e TUNES et al. (2011) para sementes de coentro, após a incubação para o teste de envelhecimento acelerado tradicional.

Tabela 2 - Valores médios obtidos para o teste de envelhecimento acelerado tradicional, a $38^{\circ} \mathrm{C}$, de lotes de sementes de quiabo (Abelmoschus esculentus (L.) Moench), cultivares 'Colhe Bem' e 'Santa Cruz 47'.

\begin{tabular}{|c|c|c|c|c|c|}
\hline \multirow{2}{*}{ Cultivar } & \multirow{2}{*}{ Lote } & \multicolumn{4}{|c|}{ Período de envelhecimento (h) } \\
\hline & & 24 & 48 & 72 & 96 \\
\hline \multirow{6}{*}{ 'Colhe Bem' } & 1 & $65 a$ & $60 a$ & $50 \mathrm{a}$ & $45 \mathrm{a}$ \\
\hline & 2 & $67 \mathrm{a}$ & $65 \mathrm{a}$ & $54 \mathrm{a}$ & $42 \mathrm{a}$ \\
\hline & 3 & $56 \mathrm{ab}$ & $55 \mathrm{ab}$ & $48 \mathrm{ab}$ & $40 \mathrm{a}$ \\
\hline & 4 & $50 \mathrm{~b}$ & $43 \mathrm{~b}$ & $40 \mathrm{~b}$ & $30 \mathrm{~b}$ \\
\hline & $\mathrm{CV}(\%)$ & 6,79 & 7,95 & 7,93 & 9,09 \\
\hline & $\mathrm{F}$ & $27,4^{*}$ & $21,6^{*}$ & $51,1^{*}$ & $26,6^{*}$ \\
\hline \multirow{7}{*}{ 'Santa Cruz 47' } & 5 & $75 \mathrm{ab}$ & $72 \mathrm{ab}$ & $72 \mathrm{a}$ & $50 \mathrm{~b}$ \\
\hline & 6 & $81 \mathrm{a}$ & $81 \mathrm{a}$ & $75 \mathrm{a}$ & $76 \mathrm{a}$ \\
\hline & 7 & $76 \mathrm{ab}$ & $72 \mathrm{ab}$ & $70 a$ & $74 \mathrm{a}$ \\
\hline & 8 & $75 \mathrm{ab}$ & $74 \mathrm{ab}$ & $72 \mathrm{a}$ & $74 \mathrm{a}$ \\
\hline & 9 & $76 \mathrm{ab}$ & $70 \mathrm{ab}$ & $68 \mathrm{a}$ & $65 \mathrm{ab}$ \\
\hline & $\mathrm{CV}(\%)$ & 11,02 & 9,01 & 9,82 & 10,65 \\
\hline & $\mathrm{F}$ & $19,6^{*}$ & $21,9^{*}$ & $2,38^{\mathrm{ns}}$ & $10,2^{*}$ \\
\hline
\end{tabular}

Médias seguidas de mesma letra na coluna, para cada cultivar, não diferem entre si pelo teste de Tukey, a $5 \%$ de probabilidade. 
Tabela 3 - Valores médios obtidos para o teste de envelhecimento acelerado tradicional, a $41^{\circ} \mathrm{C}$, de lotes de sementes de quiabo (Abelmoschus esculentus (L.) Moench), cultivares 'Colhe Bem' e 'Santa Cruz 47'.

\begin{tabular}{|c|c|c|c|c|c|}
\hline \multirow{2}{*}{ Cultivar } & \multirow{2}{*}{ Lote } & \multicolumn{4}{|c|}{--Periodo de envelhecimento (h)--- } \\
\hline & & 24 & 48 & 72 & 96 \\
\hline \multirow{7}{*}{ 'Colhe Bem' } & & 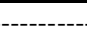 & --------- & 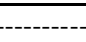 & 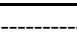 \\
\hline & 1 & $72 \mathrm{a}$ & $70 \mathrm{a}$ & $68 \mathrm{a}$ & $48 \mathrm{~b}$ \\
\hline & 2 & $69 \mathrm{a}$ & $67 \mathrm{a}$ & 67 a & $54 \mathrm{a}$ \\
\hline & 3 & $67 \mathrm{a}$ & $65 \mathrm{a}$ & $53 \mathrm{~b}$ & $46 \mathrm{~b}$ \\
\hline & 4 & $54 \mathrm{~b}$ & $52 \mathrm{~b}$ & $48 \mathrm{~b}$ & $50 \mathrm{a}$ \\
\hline & $\mathrm{CV}(\%)$ & 19,61 & 21,16 & 7,36 & 14,66 \\
\hline & $\mathrm{F}$ & $6,37^{*}$ & $4,29^{*}$ & $25,5^{*}$ & $12,3^{*}$ \\
\hline \multirow{7}{*}{ 'Santa Cruz 47' } & 5 & $73 \mathrm{a}$ & $71 \mathrm{a}$ & $59 \mathrm{ab}$ & $56 \mathrm{a}$ \\
\hline & 6 & $75 \mathrm{a}$ & $73 \mathrm{a}$ & $67 \mathrm{a}$ & $48 \mathrm{ab}$ \\
\hline & 7 & $65 \mathrm{~b}$ & $63 \mathrm{~b}$ & $52 \mathrm{~b}$ & $29 \mathrm{c}$ \\
\hline & 8 & $75 \mathrm{a}$ & $73 \mathrm{a}$ & $59 \mathrm{ab}$ & $56 a$ \\
\hline & 9 & $69 \mathrm{~b}$ & $67 \mathrm{~b}$ & $50 \mathrm{~b}$ & $32 \mathrm{c}$ \\
\hline & $\mathrm{CV}(\%)$ & 15,12 & 19,03 & 5,51 & 9,90 \\
\hline & $\mathrm{F}$ & $14,7^{*}$ & $12,9^{*}$ & $25,3^{*}$ & $16,4^{*}$ \\
\hline
\end{tabular}

Médias seguidas de mesma letra na coluna, para cada cultivar, não diferem entre si pelo teste de Tukey, a 5\% de probabilidade.

Para o grau de umidade das sementes, após o envelhecimento acelerado em solução saturada com $\mathrm{NaCl}$, em geral, os resultados foram semelhantes para os nove lotes estudados (variação de 11,0 a 14,6\%). Verificou-se que o grau de umidade das sementes expostas à solução saturada de $\mathrm{NaCl}$ apresentou valores menores e mais uniformes, após os períodos de envelhecimento em relação aos observados para as envelhecidas tradicionalmente; isso indica que o uso de solução salina contribuiu para controlar a absorção de água pelas sementes no teste de envelhecimento acelerado. Resultados semelhantes também foram

Tabela 4 - Valores médios obtidos para o teste de envelhecimento acelerado em solução saturada com $\mathrm{NaCl}$, a $38^{\circ} \mathrm{C}$, de lotes de sementes de quiabo (Abelmoschus esculentus (L.) Moench), cultivares 'Colhe Bem' e 'Santa Cruz 47'.

\begin{tabular}{|c|c|c|c|c|c|}
\hline \multirow{2}{*}{ Cultivar } & \multirow{2}{*}{ Lote } & \multicolumn{4}{|c|}{-Período de envelhecimento (h)-- } \\
\hline & & 24 & 48 & 72 & 96 \\
\hline \multirow{6}{*}{ 'Colhe Bem’ } & 1 & 56 a & $52 \mathrm{a}$ & $40 \mathrm{a}$ & $35 \mathrm{ab}$ \\
\hline & 2 & $50 \mathrm{ab}$ & $47 \mathrm{ab}$ & $41 \mathrm{a}$ & $36 \mathrm{ab}$ \\
\hline & 3 & $44 \mathrm{~b}$ & $38 \mathrm{~b}$ & $39 \mathrm{a}$ & $34 \mathrm{ab}$ \\
\hline & 4 & $51 \mathrm{ab}$ & $45 \mathrm{ab}$ & $42 \mathrm{a}$ & $44 \mathrm{a}$ \\
\hline & $\mathrm{CV}(\%)$ & 14,42 & 16,56 & 16,58 & 12,12 \\
\hline & $\mathrm{F}$ & $13,2^{*}$ & $15,9^{*}$ & $0,48^{\mathrm{ns}}$ & $4,94^{*}$ \\
\hline \multirow{7}{*}{ 'Santa Cruz 47' } & 5 & $60 \mathrm{~b}$ & $57 \mathrm{~b}$ & $51 \mathrm{~b}$ & $50 \mathrm{a}$ \\
\hline & 6 & $59 \mathrm{~b}$ & $56 \mathrm{~b}$ & $49 \mathrm{c}$ & $48 \mathrm{a}$ \\
\hline & 7 & $58 \mathrm{~b}$ & $55 \mathrm{~b}$ & $50 \mathrm{c}$ & $40 \mathrm{~b}$ \\
\hline & 8 & 73 a & $70 \mathrm{a}$ & $66 \mathrm{a}$ & $51 \mathrm{a}$ \\
\hline & 9 & $57 \mathrm{~b}$ & $54 \mathrm{~b}$ & $50 \mathrm{~b}$ & $48 \mathrm{a}$ \\
\hline & $\mathrm{CV}(\%)$ & 7,50 & 8,66 & 7,17 & 18,29 \\
\hline & $\mathrm{F}$ & $114,2^{*}$ & $126,6^{*}$ & $112,8^{*}$ & $26,2^{*}$ \\
\hline
\end{tabular}

Médias seguidas de mesma letra na coluna, para cada cultivar, não diferem entre si pelo teste de Tukey, a $5 \%$ de probabilidade.

Ciência Rural, v.44, n.12, dez, 2014. 
Tabela 5 - Valores médios obtidos para o teste de envelhecimento acelerado em solução saturada com $\mathrm{NaCl}$, a $41^{\circ} \mathrm{C}$, de lotes de sementes de quiabo (Abelmoschus esculentus (L.) Moench), cultivares 'Colhe Bem' e 'Santa Cruz 47'.

\begin{tabular}{|c|c|c|c|c|c|}
\hline \multirow{2}{*}{ Cultivar } & \multirow{2}{*}{ Lote } & \multicolumn{3}{|c|}{ Período de envelhecimento (h) } & \multirow[b]{2}{*}{96} \\
\hline & & 24 & 48 & 72 & \\
\hline \multirow{7}{*}{ 'Colhe Bem' } & & ------ & -.---- & -------- & 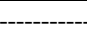 \\
\hline & 1 & $55 \mathrm{a}$ & $53 \mathrm{a}$ & $50 \mathrm{a}$ & $46 \mathrm{~b}$ \\
\hline & 2 & $57 \mathrm{a}$ & $52 \mathrm{a}$ & $50 \mathrm{a}$ & $50 \mathrm{a}$ \\
\hline & 3 & $52 \mathrm{a}$ & $50 \mathrm{a}$ & $45 \mathrm{~b}$ & $43 \mathrm{~b}$ \\
\hline & 4 & $52 \mathrm{a}$ & $50 \mathrm{a}$ & $46 \mathrm{~b}$ & $48 \mathrm{a}$ \\
\hline & $\mathrm{CV}(\%)$ & 23,22 & 26,45 & 18,14 & 6,71 \\
\hline & $\mathrm{F}$ & $3,47^{\mathrm{ns}}$ & $2,38^{\mathrm{ns}}$ & $7,92^{*}$ & $56,9^{*}$ \\
\hline \multirow{7}{*}{ 'Santa Cruz 47' } & 5 & $67 \mathrm{~b}$ & $65 \mathrm{~b}$ & $47 \mathrm{a}$ & $46 \mathrm{a}$ \\
\hline & 6 & $54 \mathrm{bc}$ & $52 \mathrm{bc}$ & $48 \mathrm{a}$ & $38 a b$ \\
\hline & 7 & $48 \mathrm{c}$ & $46 \mathrm{c}$ & $45 \mathrm{a}$ & $32 \mathrm{~b}$ \\
\hline & 8 & $72 \mathrm{a}$ & $70 \mathrm{a}$ & $40 \mathrm{ab}$ & $44 \mathrm{a}$ \\
\hline & 9 & $48 \mathrm{c}$ & $46 \mathrm{c}$ & $30 \mathrm{~b}$ & $22 \mathrm{c}$ \\
\hline & $\mathrm{CV}(\%)$ & 14,48 & 15,23 & 24,93 & 5,59 \\
\hline & $\mathrm{F}$ & $25,8^{*}$ & $34,7^{*}$ & $8,80^{*}$ & $131,3^{*}$ \\
\hline
\end{tabular}

Médias seguidas de mesma letra na coluna, para cada cultivar, não diferem entre si pelo teste de Tukey, a 5\% de probabilidade.

verificados por TUNES et al. (2011) para sementes de coentro e KIKUTI \& MARCOS FILHO (2012) para sementes de alface. Os resultados obtidos até o momento, com esse teste, demonstram que, mesmo com grau de umidade inferior, há estresse suficiente para reduzir a germinação (JIANHUA \& McDONALD, 1997; TUNES et al., 2011; KIKUTI \& MARCOS FILHO, 2012). Esse método alternativo de envelhecimento tem se revelado mais apropriado para sementes pequenas de hortaliças, como as de quiabo.

Pela análise de correlação (Tabela 6), verifica-se que os testes de envelhecimento acelerado tradicional e em solução saturada com $\mathrm{NaCl}$ por 96 horas, a $41^{\circ} \mathrm{C}$, correlacionaram-se significativamente, de forma consistente, com o teste de emergência de plântulas em campo, para ambas as cultivares. Desse modo, o teste de envelhecimento acelerado pode ser utilizado em substituição ao de emergência de plântulas em campo, para sementes de quiabo, com a vantagem de ser realizado em menor tempo. Essa ocorrência está de acordo com LIMA et al. (2011) ao informar que o teste de envelhecimento acelerado apresentou correlação altamente significativa com o de emergência de plântulas em campo, para sementes de Ocimum gratissimum. Segundo salienta MARCOS FILHO (1999), essa tendência de variação semelhante entre as duas variáveis não significa que existe a correspondente precisão de estimativa de qualidade do lote e, ainda, segundo o autor, os resultados desta análise não devem ser interpretados isoladamente. Por outro lado, TUNES et al. (2011) constataram que o teste de envelhecimento acelerado não se correlacionou com o teste de emergência em campo para sementes de coentro. Nesse sentido, MARCOS FILHO (1999) informa que a relação entre os resultados dos testes para avaliar o vigor das sementes em laboratório e o seu desempenho em campo pode, muitas vezes, ser incompatível, em função, provavelmente, dos testes de emergência de plântulas nem sempre serem adequados para detectar diferenças entre o potencial fisiológico dos lotes de sementes e, também, em razão das condições ambientais favoráveis na época da condução desse teste. O fato de haver coeficientes de correlações reduzidos e não significativos entre os diferentes testes de envelhecimento e emergência de plântulas em campo, no presente estudo, não implicam o descarte desse teste de envelhecimento para futuros trabalhos em sementes de quiabo.

Por meio da análise dos resultados, foi possível identificar que o teste de envelhecimento acelerado tradicional e em solução saturada com $\mathrm{NaCl}$, associado ao de emergência de plântulas em campo, pode ser utilizado com relativa segurança na avaliação do potencial fisiológico de sementes de quiabo, podendo variar caso as condições ambientais sejam diferenciadas das utilizadas neste estudo.

Ciência Rural, v.44, n.12, dez, 2014. 
Tabela 6 - Coeficientes de correlação simples entre os resultados dos testes de envelhecimento acelerado tradicional (EAT), envelhecimento acelerado em solução saturada com NaCl (EASS) e os resultados do teste de emergência de plântulas em campo, realizados em sementes de quiabo (Abelmoschus esculentus (L.) Moench), cultivares 'Colhe Bem' e 'Santa Cruz 47'.

\begin{tabular}{|c|c|c|c|c|}
\hline \multirow{2}{*}{ Tratamento } & & & \multirow{2}{*}{ 'Colhe Bem' } & \multirow{2}{*}{ 'Santa Cruz 47' } \\
\hline & & & & \\
\hline \multirow{9}{*}{ EAT } & \multirow{4}{*}{$38^{\circ} \mathrm{C}$} & $24 \mathrm{~h}$ & $0,52^{\mathrm{ns}}$ & $0,63^{\text {ns }}$ \\
\hline & & $48 \mathrm{~h}$ & $0,61^{\mathrm{ns}}$ & $0,50^{\mathrm{ns}}$ \\
\hline & & $72 \mathrm{~h}$ & $0,79^{\mathrm{ns}}$ & $0,65^{\mathrm{ns}}$ \\
\hline & & $96 \mathrm{~h}$ & $0,65^{*}$ & $0,55^{\mathrm{ns}}$ \\
\hline & & & & \\
\hline & \multirow{4}{*}{$41^{\circ} \mathrm{C}$} & $24 \mathrm{~h}$ & $0,75^{*}$ & $0,62^{\mathrm{ns}}$ \\
\hline & & $48 \mathrm{~h}$ & $0,70^{\mathrm{ns}}$ & $0,75^{*}$ \\
\hline & & $72 \mathrm{~h}$ & $0,67^{*}$ & $0,73^{\mathrm{ns}}$ \\
\hline & & $96 \mathrm{~h}$ & $0,98^{* *}$ & $0,99^{* *}$ \\
\hline \multirow{9}{*}{ EASS } & \multirow{4}{*}{$38^{\circ} \mathrm{C}$} & $24 \mathrm{~h}$ & $0,60^{\mathrm{ns}}$ & $0,45^{\mathrm{ns}}$ \\
\hline & & $48 \mathrm{~h}$ & $0,85^{\mathrm{ns}}$ & $0,79^{*}$ \\
\hline & & $72 \mathrm{~h}$ & $0,45^{\mathrm{ns}}$ & $0,56^{\mathrm{ns}}$ \\
\hline & & $96 \mathrm{~h}$ & $0,75^{*}$ & $0,69^{\mathrm{ns}}$ \\
\hline & & & & \\
\hline & \multirow{4}{*}{$41^{\circ} \mathrm{C}$} & $24 \mathrm{~h}$ & $0,70 *$ & $0,66^{\mathrm{ns}}$ \\
\hline & & $48 \mathrm{~h}$ & $0,88^{*}$ & $0,75^{\text {ns }}$ \\
\hline & & $72 \mathrm{~h}$ & $0,75^{\mathrm{ns}}$ & $0,72^{*}$ \\
\hline & & $96 \mathrm{~h}$ & $0,99^{* *}$ & $0,97 * *$ \\
\hline
\end{tabular}

${ }^{\text {ns }}$ não significativo, ${ }^{*}$ significativo a $1 \% \mathrm{e}^{* *}$ significativo a $5 \%$ de probabilidade.

\section{CONCLUSÃO}

Os testes de envelhecimento acelerado tradicional e em solução saturada com cloreto de sódio, a $41^{\circ} \mathrm{C}$, durante 96 horas, constituem-se em opções promissoras para detectar diferenças de vigor entre lotes de sementes de quiabo.

\section{REFERÊNCIAS}

BRASIL. Ministério da Agricultura, Pecuária e Abastecimento. Regras para análise de sementes. Ministério da Agricultura, Pecuária e Abastecimento. Secretaria de Defesa Agropecuária. Brasília, DF: Mapa/ACS, 2009. 395p.

FILGUEIRA, F.A.R. Novo manual de olericultura: agrotecnologia moderna na produção e comercialização de hortaliças. 3.ed. Viçosa: UFV, 2008. 421p.

JIANHUA, Z.; McDONALD, M.B. The saturated salt accelerated aging test for small-seeded crops. Seed Science and Technology, v.25, n.1, p.123-131, 1997.

KIKUTI, A.L.P.; MARCOS FILHO, J. Testes de vigor em sementes de alface. Horticultura Brasileira, v.30, n.1, p.44-50, 2012.

LIMA, C.B. et al. Germinação e envelhecimento acelerado na análise da qualidade fisiológica de sementes de alfavaca-cravo. Semina: Ciências Agrárias, v.32, n.3, p.865-874, 2011.
LOPES, M.M. et al. Methods for evaluating the physiological potential of scarlet eggplant (Solanum aethiopicum) seeds. Seed Science and Technology, v.40, n.1, p.86-94, 2012.

MARCOS FILHO, J. Testes de vigor: importância e utilização. In: KRZYZANOWSKI, F.C. et al. (Ed.). Vigor de sementes: conceitos e testes. Londrina: ABRATES, 1999. p.1-21.

MEDEIROS, M.A. et al. Testes de estresse térmico em sementes de melão. Revista Brasileira de Ciências Agrárias, v.9, n.1, p.7$13,2014$.

PEREIRA, M.D.; MARTINS FILHO, S. Envelhecimento acelerado em sementes de cubiu (Solanum sessiliflorum Dunal). Pesquisa Agropecuária Tropical, v.40, n.3, p.251-256, 2010.

PEREIRA, M.D. et al. Envelhecimento acelerado em sementes de pinhão-manso. Pesquisa Agropecuária Tropical, v.42, n.1, p.119-123, 2012.

PEREIRA, M.F.S. et al. Qualidade fisiológica de sementes de coentro (Coriandrum sativum L.). Revista Brasileira de Plantas Medicinais, v.13, n. esp., p.518-522, 2011.

PURQUERIO, L.F.V. et al. Germination and hardseedness of seeds in okra elite lines. Horticultura Brasileira, v.28, n.2, p.232$235,2010$.

SANTOS, F. et al. Teste de envelhecimento acelerado para avaliação da qualidade de sementes de alface e almeirão. Revista Brasileira de Sementes, v.33, n.2, p.322-323, 2011. 
TORRES, S.B.; CARVALHO, I.M.S. Teste de envelhecimento acelerado em sementes de quiabo (Abelmoschus esculentus (L.) Moench). Revista Brasileira de Sementes, v.20, n.1, p.209-211, 1998.

TORRES, S.B. et al. Controlled deterioration to evaluate okra seed vigor. Horticultura Brasileira, v.31, n.2, p.317-321, 2013.
TORRES, S.B. et al. Deterioração controlada em sementes de coentro. Revista Brasileira de Sementes, v.34, n.2, p.319-326, 2012 .

TUNES, L.M. et al. Envelhecimento acelerado modificado para sementes de coentro (Coriandrum sativum L.) e sua correlação com outros testes de vigor. Revista Brasileira de Biociências, v.9, n.1, p.12-17, 2011. 